\title{
Manufacturer Recognized a Recurring Performance Failure
}

National Cancer Institute

\section{Source}

National Cancer Institute. Manufacturer Recognized a Recurring Performance Failure. NCl Thesaurus. Code C99980.

The device manufacturer had recognized a recurrent performance failure in the device. (ACC) 